\title{
Application of Platinum Nanoparticles Decorated Three- Dimensional Graphene Modified Electrode for Methanol Electrooxidation
}

\author{
Xiaofeng Wang ${ }^{1, *}$, Xueliang Niu ${ }^{2}$, Hailiang Sha ${ }^{3}$, Fan Shi $^{2}$,Wei Chen ${ }^{2}$, Dongxue $\mathrm{Ma}^{2}$, Wei Sun ${ }^{2, *}$ \\ ${ }^{1}$ Department of Precision Instruments, Tsinghua University, Beijing 100084, P. R. China; \\ ${ }^{2}$ College of Chemistry and Chemical Engineering, Hainan Normal University, Haikou 571158, P. R. \\ China. \\ ${ }^{3}$ School of Dentistry, Capital Medical University, Beijing 100050, P. R. China; \\ *E-mail: xfw@mail.tsinghua.edu.cn, $\underline{\text { swyy26@hotmail.com }}$
}

doi: $10.20964 / 2016.09 .52$

Received: 15 June 2016 / Accepted: 13 July 2016 / Published: 7 August 2016

\begin{abstract}
A three-dimensional graphene (3DGR) was synthesized by electrodeposition directly on carbon ionic liquid electrode (CILE) with N-butylpyridinium hexafluorophosphate. Nanosized Pt catalyst was further electrodeposited onto 3DGR/CILE with its properties investigated through electron microscopy and electrochemistry. Electrocatalytic performances of the modified electrode (Pt/3DGR/CILE) to methanol oxidation were investigated. Experimental results demonstrated that an enhanced efficiency of Pt/3DGR nanohybrid on the electrode surface for methanol oxidation with the enhancement of the ratio of forward to backward peak current (If/Ib), which was due to the large surface area of nanohybrid with 3D porous structure of GR and higher dispersion of $\mathrm{Pt}$ nanoparticles onto the electrode surface.
\end{abstract}

Keywords: Three-dimensional graphene, Platinum nanoparticles, Carbon ionic liquid electrode, Methanol, Electrooxidation.

\section{$\underline{\text { FULL TEXT }}$}

(C) 2016 The Authors. Published by ESG (www.electrochemsci.org). This article is an open access article distributed under the terms and conditions of the Creative Commons Attribution license (http://creativecommons.org/licenses/by/4.0/). 